\title{
Efecto en la calidad de la información ante cambios en la normatividad contable: caso aplicado al sector real mexicano
}

\author{
Effect of information quality due accounting regulatory changes: \\ Applied case to Mexican real sector
}

\section{Héctor Horacio Garza Sánchez*, Klender Aimer Cortez Alejandro, Alma Berenice Méndez Sáenz y Martha del Pilar Rodríguez García}

Universidad Autónoma de Nuevo León, México

Recibido el 17 de marzo de 2015; aceptado el 3 de noviembre de 2015

Disponible en Internet el 17 de octubre de 2016

\section{Resumen}

El objetivo de este trabajo es analizar si los cambios en las normativas contables mejoran la relevancia valorativa de la información financiera en empresas cotizadas en México. La investigación se realizó para el periodo de 2000 a 2013 utilizando una muestra de 141 empresas que reportan a la bolsa mexicana de valores utilizando la metodología de datos panel. Nuestros hallazgos muestran que los cambios de normativa locales (principios contables generalmente aceptados) a normativas homologadas internacionalmente (normas de información financiera y normas internacionales de información financiera) aumentan la relevancia valorativa y, por consiguiente, la calidad de la información. Este estudio muestra que la información contable elaborada mediante las normas internacionales de información financiera es más confiable para los inversionistas extranjeros y nacionales.

(C) 2016 Universidad Nacional Autónoma de México, Facultad de Contaduría y Administración. Este es un artículo Open Access bajo la licencia CC BY-NC-ND (http://creativecommons.org/licenses/by-nc-nd/4.0/).

Códigos JEL: B52; C45; K11; L24

Palabras clave: Calidad de la información contable; Normas de información financiera; Normas Internacionales de Información Financiera; Principios contables

\footnotetext{
* Autor para correspondencia.

Correo electrónico: hfacpya@ hotmail.com (H.H. Garza Sánchez).

La revisión por pares es responsabilidad de la Universidad Nacional Autónoma de México.
} 


\begin{abstract}
The purpose of this paper is to examine whether changes in accounting standards improve value relevance of financial information on listed companies in Mexico. The research was conducted for the period 20002013 using a sample of 141 companies that report to the Mexican stock exchange using the methodology of panel data. Our findings show that changes in local regulations (generally accepted accounting principles) to internationally approved standards (financial reporting standards and international financial reporting standards) increase the value relevance and therefore the quality of information. The study shows that the accounting information with international financial reporting standards is more trustworthy for foreign and national investors.

(C) 2016 Universidad Nacional Autónoma de México, Facultad de Contaduría y Administración. This is an open access article under the CC BY-NC-ND license (http://creativecommons.org/licenses/by-nc-nd/4.0/).
\end{abstract}

JEL classification: B52; C45; K11; L24

Keywords: Quality of accounting information; Financial reporting standards; International Financial Reporting Standards; Accounting principles

\title{
Introducción
}

La investigación sobre la calidad de la información contable tiene un importante interés para diversos agentes, como los cuerpos emisores de normas como el Financial Accounting Standards Board (FASB) o el International Accounting Standards Board (IASB), intermediarios financieros, organismos reguladores, investigadores y académicos y, en general, para los usuarios de los estados financieros en la toma de decisiones.

Nuestro trabajo contribuye al debate de si la adopción de las normativas contables adaptadas durante el periodo de 2000 a 2013 se encuentra asociada con el incremento de la calidad de la información contable. El objetivo de este trabajo es analizar si los cambios en las normativas contables mejoran la relevancia valorativa de la información financiera en empresas cotizadas en México. Pretendemos demostrar si las variables de utilidad contable y valor contable del patrimonio neto están asociadas con el valor de mercado de las empresas enlistadas en la Bolsa Mexicana de Valores.

Nuestros hallazgos muestran que los cambios de normativa local (principios contables generalmente aceptados [PCGA]) a normativas homologadas internacionalmente (normas de información financiera $[\mathrm{NIF}]$ y normas internacionales de información financiera [NIIF]) aumentan la relevancia valorativa y, por consiguiente, la calidad de la información.

La investigación se encuentra dividida en 5 apartados. El primero corresponde a la revisión de la literatura, en el que se analizan antecedentes de normativas contables e investigaciones previas. En la segunda sección se presenta el marco teórico, en el que explicamos definiciones y mediciones de la calidad contable y de las NIIF. En la tercera sección se presenta la metodología y explicamos la muestra, los modelos a estimar y las hipótesis. Por último, la cuarta y quinta secciones corresponden a resultados y conclusiones, respectivamente.

\section{Revisión de la literatura}

\section{Antecedentes de normativas contables}

Nair y Frank (1980) señalan que en México las prácticas de contabilidad y auditoría han sido influidas por los principios generalmente aceptados en los Estados Unidos de América, con un 
enfoque orientado al establecimiento de reglas más que a principios. Sin embargo, los PCGA y las NIIF (o IFRS por sus siglas en inglés) influyen dependiendo de su contexto de aplicación (Schipper, 2003).

Los principios especifican una guía, pero requieren más juicio en su aplicación; en cambio, las reglas contemplan más requisitos, pero dejan menos espacio para la discreción (Barth et al., 2007). Con relación a las NIF, estas fueron adaptadas a las NIIF, con un enfoque más orientado a las reglas.

La actividad contable en México fue regulada en un principio por el Instituto Mexicano de Contadores Públicos, reconocido oficialmente en 1977 y encargado de emitir la normativa contable en México, en boletines y circulares de los PCGA. El Instituto Mexicano de Contadores Públicos creó el Patronato para la Investigación y Desarrollo de Normas de Información Financiera, que más tarde evolucionó al Consejo Mexicano para la Investigación y Desarrollo de Normas de Información Financiera, A.C. (CINIF), constituido en 2001 por entidades líderes de los sectores públicos y privados. No fue hasta el 2004, en congruencia con la tendencia mundial y con el objetivo fundamental de avanzar hacia una mayor convergencia con las normas de información financiera a nivel internacional, cuando asumió la función y la responsabilidad de la emisión de la normativa contable en México, llamadas NIF.

En México, la Comisión Nacional Bancaria y de Valores, procurando un desarrollo equilibrado del sistema financiero y en consideración a la evolución de los mercados globales, ha manifestado la importancia de contar con un conjunto único de normas contables a nivel internacional para lograr que la información financiera de las entidades que cotizan en dichos mercados esté determinada sobre las mismas bases y pueda usarse y compararse en cualquier parte del mundo. Por lo tanto, decidió proceder a la adaptación de las NIIF (International Financial Reporting Standards: IFRS) emitidas por el CINIF.

En 2009, la Comisión Nacional Bancaria y de Valores modificó su estrategia al adoptar directamente las normas internacionales para los ejercicios que comenzaran en 2012. En este sentido, expuso que las entidades emisoras tendrían la obligación de elaborar y divulgar su información financiera con base en las NIIF, y los estados financieros de emisoras, fideicomitentes o garantes de nacionalidad extranjera deberían ser elaborados de acuerdo con alguna de las opciones siguientes: en primer lugar, con base en las NIIF que emitiera el IASB, en segundo lugar con base en los US-GAAP, que debían incorporar en las notas complementarias correspondientes un documento explicativo de las diferencias relevantes entre las normas contables y los métodos utilizados para elaborar sus estados financieros. No obstante, aunque la fecha obligatoria fuera 1 de enero de 2012, las emisoras podían adoptarlas en forma anticipada para los ejercicios del 2008 al 2011.

Por lo anterior, podemos destacar que existen 3 periodos importantes en la aplicación de las normas contables: 1) PCGA hasta el 2005, 2) NIF en 2006-2011 y 3) NIIF de 2012 al tercer trimestre de 2013. Con estos periodos analizaremos el efecto de cada uno de ellos en la relevancia valorativa y, de esta forma, en la calidad de la información contable.

\section{Investigaciones previas}

La importancia de determinar los efectos de la incorporación de las NIIF obedece a que la información fundamental de la empresa sea de calidad para los usuarios de información financiera. Existen una gran cantidad de investigaciones sobre los efectos de la calidad contable en las variables de precios y rentabilidad, las cuales no han producido una conclusión de consenso, ya que ciertos estudios (Standifird y Weinstein, 2002; Bartov, Goldberg y Kim, 2005; Morais y Curto, 2008 y Barth, Landsman y Lang, 2008; Mohan y John, 2011; Dorantes, 2013; Karğın, 2013 y 
Bilgic y Ibis, 2013) encontraron calidad en la información financiera, mientras que en otros, los estudios presentan resultados mixtos; esto es, encuentran calidad en utilidades pero no en capital como en Agostino, Drago y Silipo (2011) y Jarva y Lantto (2012). Mientras que otros estudios no encuentran relación entre las variables contables y el valor de mercado (Davis-Friday y Rivera, 2000; Hung y Subramanyam, 2007; Morais y Curto, 2008 y Tsalavoutas, André y Evans, 2010).

Algunos estudios determinan la relevancia valorativa dividiendo la muestra en periodos prey postadopción de las NIIF. Analizan los cambios de la significación y las pendientes de las variables de las ganancias y del valor en libros. Asimismo, mencionan que si aumenta la $\mathrm{R}^{2}$ después de la adopción, existe relevancia. Entre los más importantes de este tipo de estudios están las investigaciones de Bartov et al. (2005), Morais y Curto (2008) y Barth et al. (2008).

Un estudio de empresas de 21 países que adoptan las NIIF realizado por Barth et al. (2008) concluye que las empresas que reportan bajo NIIF cuentan con una $\mathrm{R}^{2}$ significativamente mayor y exhiben una alta asociación de las cifras contables con el precio de las acciones y la rentabilidad bursátil. El mismo hallazgo se presenta en Morais y Curto (2008), que prueban que en Europa las empresas listadas presentan mayor relevancia valorativa al adoptarse las NIIF. Sin embargo, sus datos incluyen el período 2000-2005 y no distinguen entre la adopción voluntaria y la obligatoria. En Alemania, Bartov et al. (2005) evidencian que las pendientes de las utilidades basadas en NIIF tienen mayor relevancia valorativa que las pendientes de las utilidades con la normativa alemana.

Para mercados emergentes como Varsovia, Budapest y Praga, Standifird y Weinstein (2002) concluyeron que las empresas pueden mejorar su legitimidad con la adopción de la normativa contable internacional. Para el mercado mexicano, Dorantes (2013) examina la relevancia de los fundamentos de contabilidad y demuestra que sí existe relevancia valorativa de los fundamentos de contabilidad.

En India, Mohan y John, 2011 examinaron la relevancia valorativa de información y encontraron que el valor en libros por acción y utilidades por acción tienen una relación positiva estadísticamente significativa con el precio de las acciones. Utilizando esta misma metodología Karğın (2013) y Bilgic e Ibis (2013) encontraron que el valor en libros es determinante para el precio de la acción y el valor de mercado en Estambul. Rawashdeh (2003) para el mercado de Jordania encontró un impacto significativo en el precio de las acciones tras el cambio en la normativa contable, lo cual provee una mayor relevancia valorativa al mercado, con resultados más significativos en las empresas de menor tamaño.

Por otra parte, encontramos estudios con resultados mixtos, ya que existe relevancia en ingresos, pero no en capital. Agostino et al. (2011) en su estudio para 15 bancos europeos encuentran que el efecto marginal (relevancia valor) de los ingresos aumentó para toda la muestra, mientras que no pasaba lo mismo con la variable del capital, cuyo efecto es negativo al introducirse la norma internacional. Además, incluyeron una variable dummie determinante, que es cero cuando la NIIF no es obligatoria y uno cuando sí lo es.

En Jarva y Lantto (2012) para empresas listadas en Finlandia encontraron que las ganancias bajo la NIIF tienen mayor efecto en el valor de mercado de la empresa que bajo la normativa local. Asimismo, concluyen que el valor en libros medidos bajo NIIF no tiene mayor relevancia valorativa que en aquellas empresas que reportaron bajo normativa local y, por consiguiente, no son de mayor calidad. Esto pudiera deberse a la capacidad explicativa de las ganancias, las cuales son una medida contable de la rentabilidad que mide la capacidad de una empresa para hacer futuros pagos de dividendos (Morais y Curto, 2008).

Por último, en algunas investigaciones encontramos que las NIIF no han afectado a la calidad de la información. Tsalavoutas et al. (2010) concluyen que no encuentran cambios significativos en la relevancia valorativa del valor en libros del capital y los resultados en estos 2 períodos, en un 
ambiente con débil gobierno corporativo como el mercado griego. Hung y Subramanyam (2007) encontraron poca evidencia de que las NIIF mejoren la relevancia valorativa en el valor de los libros y en el resultado neto. Davis-Friday y Rivera (2000) indican que el reconciliar información contable bajo normas locales con americanas no tiene un impacto significativo en el precio de la acción.

Morais y Curto (2008), para una muestra de empresas portuguesas, concluyen que cambiar los periodos de adopción de las normas locales portuguesas a normas internacionales no mejora el efecto sobre el precio de la acción de las ganancias por acción y del valor en libros de la acción. Asimismo, encuentran que la $\mathrm{R}^{2}$ disminuye en el periodo de implementación de las NIIF.

Estos hallazgos son contradictorios con la literatura previa, que muestra que la adopción de las NIIF mejora la relevancia de valor. Sin embargo, estos resultados pueden justificarse, ya que el entorno político y económico puede afectar en las prácticas de presentación de informes financieros del país (Ball, 2006).

Las investigaciones mencionadas anteriormente están basadas en la hipótesis de mercado eficiente, la cual afirma que los mercados reaccionan racionalmente a la llegada de nueva información y los precios varían a medida que esta se recibe. Existen 3 formas de eficiencia en precios, como señala Fama (1970): débil, fuerte y semifuerte. En esta investigación se asume esta última, que supone que los precios de hoy reflejan toda la información pública accesible como dividendos, información fiscal y financiera, noticias e información del estatus de la economía.

\section{Marco teórico}

\section{Calidad de la información contable}

La información contable es utilizada para entender la realidad económica de la empresa con la finalidad de tomar decisiones adecuadas, por lo que se debe definir a través de la calidad de esta (Dumitru, 2011). Dicha calidad de la información contable ha estado recibiendo mayor atención debido a los escándalos contables recientes. Sin embargo, a pesar del incremento de la importancia de este tema, la calidad de la contabilidad sigue siendo un término vago y difícil de definir (Bartov et al., 2005; Hribar, Kravel y Wilson, 2014).

Existe una gran cantidad de definiciones sobre el concepto de calidad de la información contable. Desde una aproximación cuantitativa, destacan los trabajos de Penman y Zhang (2002), Dechow y Schrand (2004) y Dechow, Ge y Schrand (2010), quienes definen la alta calidad a través del efecto predictivo de la variable de utilidad contable sobre la valoración futura de la empresa. Por otra parte, Barth et al. (2008) definen que existe calidad del resultado cuando se presenta una menor manipulación de la información contable, un reconocimiento más oportuno de las pérdidas así como un incremento en la capacidad predictiva dada por la regresión entre las variables fundamentales y las de mercado.

Dechow et al. (2010) mencionan que no hay una medida de la calidad para todos los modelos de decisión. Otros autores como Ball, Robin y Wu (2003), Ball y Shivakumar (2005) y Burgstahler, Hail y Leuz (2006) afirman que la calidad de la información contable varía conforme se incrementa el número de usuarios que tienen acceso a la información privilegiada, debido a que resuelve la asimetría de la información en las empresas privadas.

A pesar de la inexistencia de una clara definición de la calidad de la contabilidad, varios estudios usan medidas que se consideran sustitutos de calidad contable, por ejemplo, la administración de las ganancias, el reconocimiento oportuno de las pérdidas y la relevancia valorativa (Barth et al., 2008). En nuestra investigación consideramos la relevancia valorativa para medir la calidad de 
la información como en Francis, LaFond, Olsson y Schipper (2004) y Agostino et al. (2011). La investigación en relevancia valorativa se ha realizado fundamentalmente en países desarrollados. Sin embargo, existe una escasa evidencia en países en desarrollo y en particular en América Latina, y México no es la excepción.

\section{Normas internacionales de información financiera}

Las NIIF son normas contables emitidas por el Consejo de Normas Internacionales de Contabilidad (IASB), una organización independiente con sede en Londres (Reino Unido). Pretenden ser un conjunto de reglas que, idealmente, se aplicaría igualmente a los informes financieros por las empresas públicas de todo el mundo. Entre 1973 y 2000 las normas internacionales fueron emitidas por la organización predecesora de la IASB, el Comité de Normas Internacionales de Contabilidad (IASC). Este es un órgano creado en 1973 por asociaciones profesionales de contadores en Australia, Canadá, Francia, Alemania, Japón, México, Países Bajos, Reino Unido e Irlanda y los Estados Unidos. Durante ese período, las reglas del IASC fueron descritas como «International Accounting Standards» (IAS).

Desde abril de 2001, esta función de elaboración de normas ha sido tomada por un reconstituido IASB. El IASB describe sus reglas bajo la nueva etiqueta «International Financial Reporting Standards» (IFRS), a pesar de que continúa reconociendo (acepta como legítimas) las normas anteriores (IAS) emitidas por el antiguo organismo normativo (IASC). El IASB está mejor financiado, cuenta con mejor personal y es más independiente que su predecesor.

Para Ball (2006) existen 3 ventajas al adoptar las NIIF. En primer lugar, producen economías de escala, ya que solo se inventan una vez: serían un tipo de bien público, el costo marginal de aplicarse a una nueva empresa es cero. La segunda ventaja es que protegen a los auditores contra la manipulación de la información de los administradores y la tercera es que permite la comparación de información entre diferentes países. Esta tercera ventaja facilita la inversión transfronteriza y la integración del mercado de capitales (Aggarwal, Klapper y Wysocki, 2005).

En la última década ha habido una creciente atención sobre cómo los informes corporativos pueden mitigar los problemas de agencia y la falta de información. La literatura (Ball et al., 2003; Rawashdeh, 2003; Armstrong, Barth y Riedl, 2010) ha tratado de responder a cuestiones tales como si las normas internacionales producen información relevante o de calidad para los inversionistas y partes interesadas. Sobre esta cuestión, para Barth et al. (2008) las NIIF son normas de mejor calidad que las nacionales, que podrían implicar un «efecto reputacional» para las empresas que las adopten de manera voluntaria.

Aunque quizá puedan existir diferencias en la aplicación de las NIIF derivadas de cuestiones de transparencia y de su obligatoriedad. Daske, Hail, Leuz y Verdi (2007) encontraron que los efectos económicos de la adopción de las NIIF dependen de la medida de un serio compromiso con la transparencia. Asimismo, Daske, Hail, Leuz y Verdi (2008) concluyeron que los efectos del mercado de capitales ante los cambios de las NIIF cuando estas son obligatorias solo se producen en países con regímenes estrictos y donde el entorno institucional proporciona fuertes incentivos para que las empresas sean transparentes.

\section{Metodología}

La muestra está compuesta por 141 empresas emisoras de la Bolsa Mexicana de Valores durante el periodo de 2000 al 2013, considerando solo el sector real. A efectos de este estudio 
hemos consultado la base de datos de Economática para obtener las series trimestrales de variables contables y de mercado.

En esta investigación pretendemos contrastar si las variables contables fundamentales tienen relevancia valorativa y, por consiguiente, calidad financiera en el mercado bursátil en México. En primer lugar deseamos conocer si existe calidad financiera en el mercado mexicano durante el periodo de estudio (2000-2013). Para ello con base en los estudios de Mohan y John (2011) y Dorantes (2013), determinaremos la significación en beneficio antes de intereses y de impuestos (BAII) y de patrimonio neto de la empresa (PAT). Las hipótesis quedarían como:

$H_{a}^{1}$ : Las variables fundamentales (BAII y PAT) tienen un impacto en la capitalización de la empresa.

Además, determinamos si las NIIF medidas como una variable dicotómica tienen impacto en la capitalización, como lo determinan Agostino et al. (2011) y Cameran, Campa y Pettinicchio (2014) en su estudio. De esta forma nuestra segunda hipótesis quedaría como:

$H_{a}^{2}$ : El efecto de las NIIF es mayor que el de los PCGA y el efecto de las NIF es mayor que el de los PCGA.

Por otra parte, para ser más contundentes con nuestros resultados dividimos la muestra en pre- y postadopción de las NIIF como en Bartov et al. (2005), Morais y Curto (2008) y Barth et al. (2008). Además, demostraremos si existen aumentos en la $\mathrm{R}^{2}$, para comprobar esto, nuestra tercera y cuarta hipótesis quedarían como:

$H_{a}^{3}$ : El BAII y el PAT aumentan su significación y valor al cambiar los periodos de estudio de PCGA a NIF y a NIIF.

$H_{a}^{4}$ : La $\mathrm{R}^{2}$ aumenta al pasar de un periodo de normas locales (PCGA y NIF) a un periodo de normas internacionales NIIF.

Para probar las primeras 2 hipótesis utilizamos todo el periodo de estudio, esto es, 2000 a 2013 y para la tercera y cuarta hipótesis identificamos 3 periodos: 1) PCGA del 2000 al 2005, 2) NIF de 2006 a 2011 y 3) IFRS de 2012 al tercer trimestre de 2013. Para este último caso se eliminaron las variables dummies.

Para probar el primer caso, esto es la totalidad de la muestra, estimamos el modelo de la ecuación (1).

$$
\begin{aligned}
\text { Cap }_{i t}= & \beta_{0}+\beta_{1} \text { PAT }_{i t}+\beta_{2} \text { BAII }_{i t}+\beta_{3} \text { Rot_Activo }_{i t}+\beta_{4} \text { Tamañ }_{i t} \\
& +\beta_{5} \text { Rot_Deuda }_{i t}+\beta_{6} \text { Crec }_{i t}+\beta_{7} N I F+\beta_{8} N I I F+e_{i t}
\end{aligned}
$$

Donde:

$C a p_{i t}=$ Valor de mercado $^{1}$ de la empresa $i$ del período $t$ deflactado con el activo del periodo $t-1$.

$P A T_{i t}=$ Patrimonio neto de la empresa $i$ en el momento $t$ deflactado con el activo del periodo $t-1$.

$B A I I_{i t}=$ Beneficio antes de intereses e impuestos de la empresa $i$ en el momento $t$ deflactado con el activo del periodo $t-1$.

Rot_Activo ${ }_{i t}=$ Ventas totales entre activo total de la empresa $i$ en el periodo $t$.

Tamaño $_{i t}=$ Logaritmo natural del activo de la empresa $i$ en el periodo $t$.

\footnotetext{
${ }^{1}$ Para el cálculo del valor de mercado se multiplica el precio de las acciones por el número de acciones en circulación de cada emisora.
} 
Rot_Deuda $a_{i t}=$ Tasa de cambio anual del pasivo total de la empresa $i$ del periodo $t$ con respecto al periodo $t$ - 1 , utilizando primeras diferencias logarítmicas.

$\mathrm{Crec}_{t}=$ Tasa de cambio anual de las ventas de la empresa $i$ del periodo $t$ con respecto al periodo $t-1$, utilizando primeras diferencias logarítmicas.

$N I F_{t}=$ Variable dicotómica que toma el valor de uno si los estados financieros de la empresa $i$ están elaborados en el periodo $t$ con normas adaptadas a las NIF y 0 en caso contrario.

$N I I F_{t}=$ Variable dicotómica que toma el valor uno si los estados financieros de la empresa $i$ están elaborados en el periodo $t$ con normas adoptadas a las NIIF y 0 en caso contrario.

Siguiendo los estudios de Karğın (2013), Bilgic e Ibis, 2013, Agostino et al. (2011) y Kothari y Zimmerman (1995), consideramos la capitalización como variable dependiente. Para las variables independientes seleccionamos como variables contables fundamentales el BAII y el patrimonio (Ohlson, 1995; Dechow y Schrand, 2004; Dechow et al., 2010). Para la variable de eficiencia, siguiendo a Lang, Smith Raedy y Wilson (2006), incorporamos la variable de rotación de activos, la cual se mide por el cambio de los activos anualizado.

Como variables de control consideramos el tamaño de la empresa determinado por el logaritmo natural del activo total siguiendo a Watts (2003), Francis et al. (2004), Ball y Shivakumar (2005) y Khan y Watts (2009). Por otra parte, incluimos la rotación de deuda medida a través de la variación del pasivo (Nikolaev, 2010; Paananen y Lin, 2009; Lang et al., 2006; Barth et al., 2008; Ahmed, Neel y Wang, 2013; Chen, Tang, Jiang y Lin, 2010).

Controlamos el efecto anticipado de los resultados futuros en el crecimiento de las empresas a través de la variable crecimiento, medido por la tasa de variación de las ventas. En este sentido, Khan y Watts (2009) sostienen que las empresas de alto crecimiento muestran más volatilidad en el rendimiento de las acciones, son más propensas a tener pérdidas muy grandes y, por lo tanto, afirman que existe una relación positiva entre esta variable y el precio de la acción. Al igual que nuestras variables fundamentales, esperamos que las variables de control tengan una mayor relación con la variable de mercado a medida que mejora la calidad de la información.

Por otra parte, consideramos todos los periodos de la muestra para generalizar los resultados, incorporamos 2 variables dicotómicas, la primera (NIF) que toma el valor uno si los estados financieros están elaborados con normas adaptadas a las NIF y 0 en caso contrario, y la segunda variable (NIIF) que toma el valor uno si los estados financieros están elaborados con normas adoptadas a las NIIF y 0 en caso contrario.

Debido a que las normas contables para el caso mexicano han ido homologándose hacia un contexto internacional, pretendemos probar si la relevancia valorativa mejora a través del tiempo. Para ello, estimamos 3 modelos divididos a través de la aplicación de las diferentes normas que se han aplicado en México, para lo cual identificamos 3 periodos: 1) PCGA del 2000 al 2005, 2) NIF del 2006 al 2011 y 3) NIIF del 2012 al tercer trimestre de 2013. El modelo propuesto se presenta a través de la siguiente ecuación:

$$
\begin{aligned}
\text { Cap }_{i t}= & \beta_{0}+\beta_{1} \text { PAT }_{i t}+\beta_{2} \text { BAII }_{i t}+\beta_{3} \text { Rot_Activo }_{i t}+\beta_{4} \text { Tamañ }_{i t} \\
& +\beta_{5} \text { Rot_Deuda }_{i t}+\beta_{6} \text { Crec }_{i t}+e_{i t}
\end{aligned}
$$

Contrastaremos nuestras hipótesis con la metodología de datos de paneles, utilizando el software Eviews para datos panel. Debido a la heterogeneidad de nuestras observaciones, en relación con las empresas asumimos la presencia de heterocedasticidad en este sentido y, por ello, empleamos mínimos cuadrados generalizados. Asimismo, para probar la relevancia contable consideramos si el estadístico $\mathrm{R}^{2}$ aumenta a través del tiempo. 
Tabla 1

Coeficientes de correlación de las variables

\begin{tabular}{lcccccr}
\hline & BAII & PAT & Tamaño & Rot_Activo & Crec & Rot_Deuda \\
\hline BAII & 1.000 & 0.270 & 0.283 & 0.267 & 0.151 & 0.124 \\
PAT & 0.270 & 1.000 & -0.042 & 0.013 & 0.101 & 0.108 \\
Tamaño & 0.283 & -0.042 & 1.000 & 0.006 & 0.109 & 0.069 \\
Rot_Activo & 0.267 & 0.013 & 0.006 & 1.000 & 0.078 & -0.022 \\
Crec & 0.151 & 0.101 & 0.109 & 0.078 & 1.000 & 0.374 \\
Rot_Deuda & 0.124 & 0.108 & 0.069 & -0.022 & 0.374 & 1.000 \\
\hline
\end{tabular}

BAII: beneficio antes de intereses e impuestos de la empresa $i$ en el momento $t$ deflactado con el activo del periodo $t$ - 1 ; PAT: patrimonio neto de la empresa $i$ en el momento $t$ deflactado con el activo del periodo $t$ - 1 . Crec: tasa de cambio anual de las ventas de la empresa $i$ del periodo $t$ con respecto al periodo $t$ - 1 , utilizando primeras diferencias logarítmicas. Rot_Activo: ventas totales entre activo total de la empresa $i$ en el periodo $t$; Rot_Deuda: tasa de cambio anual del pasivo total de la empresa $i$ del periodo $t$ con respecto al periodo $t$ - 1 , utilizando primeras diferencias logarítmicas.

Fuente: Elaboración propia utilizando Eviews 7.

Tabla 2

Estimación de la muestra para 2000-2013

\begin{tabular}{|c|c|c|c|c|}
\hline Cap & Coeficientes & Error estándar & Estadístico t & $\mathrm{p}$ \\
\hline BAII & 2.1839 & 0.3672 & 5.9472 & 0.0000 \\
\hline PAT & 1.1531 & 0.1077 & 10.7074 & 0.0000 \\
\hline Tamaño & 8.5837 & 1.2619 & 6.8019 & 0.0000 \\
\hline Rot_Activo & 0.1297 & 0.0389 & 3.3257 & 0.0009 \\
\hline Crec & 0.1626 & 0.0560 & 2.9010 & 0.0037 \\
\hline Rot_Deuda & 0.1783 & 0.0472 & 3.7730 & 0.0002 \\
\hline NIIF & 41.3077 & 5.5656 & 7.4218 & 0.0000 \\
\hline NIF & 28.8802 & 3.6747 & 7.8591 & 0.0000 \\
\hline Constante & -158.5610 & 20.7991 & -7.6234 & 0.0000 \\
\hline $\mathrm{R}^{2}$ & 0.5619 & & $\mathrm{~N}$ & 3,231 \\
\hline
\end{tabular}

BAII: beneficio antes de intereses e impuestos de la empresa $i$ en el momento $t$ deflactado con el activo del periodo $t$ - 1 ; PAT: patrimonio neto de la empresa $i$ en el momento $t$ deflactado con el activo del periodo $t$ - 1 ; Crec: tasa de cambio anual de las ventas de la empresa $i$ del periodo $t$ con respecto al periodo $t$ - 1 , utilizando primeras diferencias logarítmicas; Rot_Activo: ventas totales entre activo total de la empresa $i$ en el periodo $t$; Rot_Deuda: tasa de cambio anual del pasivo total de la empresa $i$ del periodo $t$ con respecto al periodo $t$-1, utilizando primeras diferencias logarítmicas; NIF: normas de información financiera; NIIF: normas internacionales de información financiera; $\mathrm{R}^{2}$ : R cuadrada.

Fuente: Elaboración propia utilizando Eviews 7.

\section{Resultados}

Considerando el periodo de estudio 2000-2013 para 141 unidades, se calculó la correlación entre las variables independientes con el fin de detectar una posible multicolinealidad entre las variables. Los valores son relativamente pequeños, lo que indica una baja correlación entre las variables, es decir, no hay indicios de multicolinealidad (tabla 1).

En la tabla 2 se muestran los resultados del análisis del modelo de datos panel ${ }^{2}$. Como se puede observar, todas las variables fundamentales del modelo son positivas y significativas con un $95 \%$

\footnotetext{
${ }^{2}$ Se utilizó la corrección de White period standard errors \& covariance que es una herramienta diseñada para arreglar la correlación serial y las variaciones en el tiempo de los errores, comunes en los datos panel.
} 
Tabla 3

Modelos para cada uno de los periodos

\begin{tabular}{|c|c|c|c|c|c|c|}
\hline & \multicolumn{2}{|c|}{ PCGA } & \multicolumn{2}{|c|}{ NIF } & \multicolumn{2}{|c|}{ NIIF } \\
\hline & Coeficiente & $\mathrm{p}$ & Coeficiente & $\mathrm{p}$ & Coeficiente & $\mathrm{p}$ \\
\hline BAII & 0.3294 & 0.3666 & 3.6411 & 0.0000 & 3.0880 & 0.0000 \\
\hline$P A T$ & 0.5764 & 0.0000 & 1.2558 & 0.0000 & 1.7742 & 0.0000 \\
\hline Tamaño & 2.6754 & 0.0072 & 7.2089 & 0.0000 & 11.3044 & 0.0021 \\
\hline Rot_Activo & 0.0941 & 0.0208 & 0.0510 & 0.1097 & 0.1531 & 0.0000 \\
\hline Crec & 0.3317 & 0.0004 & 0.1207 & 0.0012 & 0.0896 & 0.0908 \\
\hline Rot_Deuda & 0.1589 & 0.0135 & 0.0000 & 0.0021 & 0.2194 & 0.0024 \\
\hline Constante & -34.7371 & 0.0431 & -115.7754 & 0.0000 & -195.8749 & 0.0000 \\
\hline$R^{2}$ & \multicolumn{2}{|c|}{0.4992} & \multicolumn{2}{|c|}{0.5654} & \multicolumn{2}{|c|}{0.8188} \\
\hline $\mathrm{N}$ & \multicolumn{2}{|c|}{713} & \multicolumn{2}{|c|}{1,961} & \multicolumn{2}{|c|}{557} \\
\hline
\end{tabular}

BAII: beneficio antes de intereses e impuestos de la empresa $i$ en el momento $t$ deflactado con el activo del periodo $t$ - 1 ; PAT: patrimonio neto de la empresa $i$ en el momento $t$ deflactado con el activo del periodo $t$ - 1 ; Crec: tasa de cambio anual de las ventas de la empresa $i$ del periodo $t$ con respecto al periodo $t$ - 1 , utilizando primeras diferencias logarítmicas; Rot_Activo: ventas totales entre activo total de la empresa $i$ en el periodo $t$; Rot_Deuda: tasa de cambio anual del pasivo total de la empresa $i$ del periodo $t$ con respecto al periodo $t$-1, utilizando primeras diferencias logarítmicas; NIF: normas de información financiera; NIIF: normas internacionales de información financiera; N: número de empresas; PCGA: principios contables generalmente aceptados; $\mathrm{R}^{2}$ : $\mathrm{R}$ cuadrada.

Fuente: Elaboración propia utilizando Eviews 7.

de confianza ${ }^{3}$, con ello probamos la primera hipótesis. Respecto a las variables dicotómicas, se encontró que son estadísticamente significativas, lo que confirma que existe un incremento en la relevancia de la información contable al cambiar las normas.

Con relación al impacto de la entrada en vigor de las NIF vs. NIIF, encontramos que el coeficiente de las NIIF es de 41.3 en comparación con los PCGA. Por otro lado, el coeficiente de las NIF es de 28.8 mayor que los $\mathrm{PCGA}^{4}$. En términos generales, dado este hallazgo, se interpreta que las NIIF tienen un impacto mayor que las NIF, considerando el coeficiente estimado para estas variables en el modelo, y, a su vez, estos 2 tienen mayor impacto que los PCGA. Estos resultados revelan la importancia que le otorga el mercado a la implementación de normas homologadas bajo un contexto internacional.

Nuestros resultados son congruentes con Rawashdeh (2003), Bartov et al. (2005) y Barth et al. (2008), que encuentran que tras normativas de mayor calidad se incrementa la relevancia valorativa de la información contable, y contrarios a Hung y Subramanyam (2007) y Tsalavoutas et al. (2010). Podemos argumentar que, a partir de los resultados, las empresas públicas en México se han beneficiado con una mayor reputación al cambiar de normas locales a internacionales. Los efectos en el mercado de capitales serían un mayor capital de inversionistas extranjeros que buscan empresas que revelan la información financiera según estándares internacionales, y que mitigan de esta forma los problemas de transparencia y de falta de información.

\footnotetext{
${ }^{3}$ Se consideraron estimación de modelos con efectos fijos y aleatorios siendo los efectos fijos en unidades de corte transversal los más apropiados bajo la prueba de Hausmann. Sin embargo que los resultados no fueron muy diferentes a los presentados en este artículo y las variables de control tamaño y Rot_Activo no significativas, por lo que se optó por considerar el modelo de datos panel considerando datos panel con la corrección de pesos en corte transversal.

${ }^{4}$ Este resultado se validó aplicando la prueba de Wald considerando la siguiente hipótesis Ho: $B(N I I F)-B(N I F)=0$, la cual fue rechazada.
} 
Tabla 4

Modelos para los primeros 7 trimestres de la normativa

\begin{tabular}{|c|c|c|c|c|}
\hline & \multicolumn{2}{|c|}{ NIF } & \multicolumn{2}{|c|}{ NIIF } \\
\hline & Coeficiente & $\mathrm{p}$ & Coeficiente & $\mathrm{p}$ \\
\hline$B A I I$ & 3.7567 & 0.0000 & 3.0880 & 0.0000 \\
\hline$P A T$ & 1.0727 & 0.0000 & 1.7742 & 0.0000 \\
\hline Tamaño & 7.5355 & 0.0000 & 11.3044 & 0.0000 \\
\hline Rot_Activo & 0.1063 & 0.0510 & 0.1531 & 0.0021 \\
\hline $\mathrm{Crec}$ & 0.1575 & 0.1207 & 0.0896 & 0.0908 \\
\hline Rot_Deuda & 0.5242 & 0.0000 & 0.2194 & 0.0024 \\
\hline Constante & -112.8425 & 0.0000 & -195.8749 & 0.0000 \\
\hline$R^{2}$ & \multicolumn{2}{|c|}{0.6781} & \multicolumn{2}{|c|}{0.8188} \\
\hline $\mathrm{N}$ & \multicolumn{2}{|c|}{596} & \multicolumn{2}{|c|}{557} \\
\hline
\end{tabular}

BAII: beneficio antes de intereses e impuestos de la empresa $i$ en el momento $t$ deflactado con el activo del periodo $t$ - 1 ; PAT: patrimonio neto de la empresa $i$ en el momento $t$ deflactado con el activo del periodo $t$ - 1 ; Crec: tasa de cambio anual de las ventas de la empresa $i$ del periodo $t$ con respecto al periodo $t$ - 1 , utilizando primeras diferencias logarítmicas; Rot_Activo: ventas totales entre activo total de la empresa $i$ en el periodo $t$; Rot_Deuda: tasa de cambio anual del pasivo total de la empresa $i$ del periodo $t$ con respecto al periodo $t-1$, utilizando primeras diferencias logarítmicas; NIF: normas de información financiera; NIIF: normas internacionales de información financiera; N: número de empresas; $\mathrm{R}^{2}$ : R cuadrada. Fuente: Elaboración propia utilizando Eviews 7.

Para validar la tercera y cuarta hipótesis consideramos el modelo de la ecuación (2) para cada uno de los periodos con el propósito de analizar cada uno por separado. Los estudios de Rawashdeh (2003) y Agostino et al. (2011) proponen dividir la muestra en varios periodos considerando el cambio de normativa: si hay incremento en los coeficientes de las variables fundamentales como BAII y patrimonio, se prueba el impacto del cambio normativa, ya que en principio estaría mejorando la relevancia de información contable; si además se incrementa la $\mathrm{R}^{2}$ se apoyaría aún más la hipótesis de que la relevancia mejora.

Los resultados se muestran en la tabla 3. Las variables contables fundamentales patrimonio neto y beneficios son positivas y significativas, considerando un $95 \%$ de confianza en los 3 periodos. En relación con las variables de control, todas son significativas al 90 y $95 \%$ de importancia, a excepción de la variable rotación de activos para el periodo de las NIF, como los hallazgos de Paananen y Lin (2009), los cuales no encuentran evidencia en esta variable. Por tanto, obtenemos evidencia de que los inversionistas valoran la información contenida en los estados financieros en México. Nuestros resultados son congruentes con Bartov et al. (2005) y Barth et al. (2008).

Los resultados también muestran que se incrementa la relevancia valorativa tras la adopción de las NIF. Lo anterior es debido a que, al pasar del periodo de los PCGA a las NIF, la $\mathrm{R}^{2}$ tiene un incremento del 14\%, lo que nos indica una mejora en la relevancia valorativa. Con relación al impacto de las variables fundamentales podemos observar que solo el $50 \%$ de los coeficientes estimados se incrementaron durante estos 2 periodos, sin embargo, las 2 variables fundamentales (BAII y PAT) aumentaron. Esto nos indica que mejora la calidad de la información, mejora la capacidad predictiva de las variables fundamentales (Barth et al., 2008) y de esta forma disminuyen los problemas de asimetría de la información (Ball et al., 2003; Ball y Shivakumar, 2005; y Burgstahler et al., 2006)

En el caso del cambio de las NIF a las NIIF encontramos que la $\mathrm{R}^{2}$ también aumenta, pero su crecimiento fue mayor en un $44 \%$. Lo anterior indica que la adopción de normas internacionales mejora la calidad de la información contable. Sin embargo, el periodo de análisis considerado en las NIIF es pequeño en contraste con las NIF. Por ello, se aplicó nuevamente el modelo para 
el caso de las NIF, teniendo en cuenta el impacto solo al inicio de la adopción, esto es, solo los primeros 7 trimestres para poder compararlo. Los resultados se muestran en la tabla 4.

La $\mathrm{R}^{2}$ en el periodo de las NIF aumentó si solo consideramos los primeros 7 trimestres, pero aun así la $\mathrm{R}^{2}$ en el modelo de las NIIF fue mayor, es decir, el impacto del inicio de las NIIF fue mayor que en las NIF con relación a la calidad de la información contable. Respecto al impacto de las 2 variables fundamentales, el PAT aumentó mientras que el BAII disminuyó, aunque este descenso solo fue del $17 \%$. Una explicación de lo anterior podría tener relación con el entorno financiero externo, ya que durante los primeros 7 trimestres del periodo de las NIF los resultados de las empresas no reflejaban la crisis financiera internacional del 2008 ni la situación económica de Europa en los periodos posteriores. Al respecto, Davis-Friday y Gordon (2002) señalan que el patrimonio neto conserva su significado y poder explicativo durante la crisis, y que los beneficios dejan de presentar contenido informativo, atribuido a la presencia de resultados negativos.

\section{Conclusiones}

El mercado valora de forma distinta según la normativa empleada en la elaboración de la información contable. Determinamos la calidad de la normativa contable medida por un incremento en la relevancia valorativa de la información financiera. En este sentido, la confianza en las normas contables se ha incrementado a partir de 2006, año en el que se incorporan las NIF y posteriormente en 2012, cuando se adoptan las NIIF.

Este estudio ofrece contribuciones importantes para el mercado mexicano. En primer lugar, los inversionistas extranjeros y nacionales tienen cierto escepticismo sobre la transparencia de las empresas al divulgar su información contable y, por otra parte, sobre las instituciones encargadas de vigilar la adopción de las reglas contables (Dorantes, 2013). En este sentido nuestros resultados demuestran que los coeficientes de las variables fundamentales y de control son significativos, además las 2 variables dicotómicas (NIF e NIIF) también son significativas y positivas, y el coeficiente de la variable del NIIF es mayor que el coeficiente de la NIF. Esto demuestra que los cambios en la normativa contable tienen impacto sobre la calidad de la información durante el periodo del estudio 2000-2013. El periodo de NIIF arroja mayor relevancia que el periodo de NIF. Con estos resultados se ofrece a los inversionistas señales de tranquilidad con relación a nuestras instituciones y a la transparencia de nuestras empresas.

En segundo lugar, con el fin de mostrar una mayor contundencia en nuestros resultados y valorar la adaptación de las NIF a las NIIF, optamos por recortar el periodo en estos 2 casos con el fin de encontrar el efecto en los 7 trimestres después de la adopción de cada una de estas normas. Nuestros resultados arrojan que el cambio de mayor impacto en la relevancia valorativa se produce cuando se adaptan las NIIF, medido a través del incremento en los coeficientes de las variables fundamentales y de la capacidad predictiva del modelo $\left(\mathrm{R}^{2}\right)$, lo que demuestra que el trabajo del CINIF ha sido efectivo. Nuestros resultados son similares a los obtenidos por Bartov et al. (2005) y Barth et al. (2008).

En tercer lugar, sobre las ventajas de la incorporación de las NIIF con base en Ball (2006) y que se pudieron transferir al mercado mexicano, según nuestros hallazgos podría ser que los inversores pudieran disfrutar de información más precisa, completa y oportuna que les permita tener menos asimetría de información y con ello disminuir los problemas de riesgo. Además, la incorporación de las NIIF mejora el procesamiento de la información, lo que mejora la eficiencia en los mercados y el reflejo de la información contable en los precios de las acciones. También reduce las diferencias internacionales de comparación, lo que pudiera eliminar barreras para adquisiciones y desinversiones y recompensaría a los inversores con un aumento en las primas de adquisición. 
En cuarto lugar, las NIIF permiten mayor calidad de la información contable, lo que mejora la transparencia y el gobierno corporativo. Debido a que las NIIF se establecen con base en reglas (Barth et al., 2007), los administradores están más controlados para manipular información y pudieran presentarse menos problemas de agencia, todo esto en beneficio de los accionistas.

En quinto lugar, el reconocimiento de las pérdidas de forma oportuna provoca que los administradores puedan contar con información con más rapidez sobre las inversiones que están generando pérdidas $\mathrm{y}$, de esta manera, pueden orientarse a inversiones que generen VPN positivos (Ball y Shivakumar, 2005).

Por último, nuestros hallazgos prueban la necesidad de dedicar esfuerzos adicionales para lograr un juego único de normas contables admitidas en los mercados de capitales a nivel internacional con el fin de darle sustentabilidad a la transparencia que han logrado tanto las NIF como las NIIF. Lo anterior nos permite concluir 2 cuestiones, por un lado, los inversionistas pueden confiar en la información contable como criterio de inversión y, por otro lado, los administradores de las empresas deben concientizarse de que las variables fundamentales de la empresas pueden beneficiar o perjudicar el desempeño de su mercado, por lo cual se necesita que las empresas cuenten con desempeños financieros sostenibles en el tiempo.

\section{Referencias}

Ahmed, A. S., Neel, M. J. y Wang, D. D. (2013). Does mandatory adoption of IFRS improve accounting quality? Preliminary evidence. Contemporary Accounting Research, 30(4), 1344-1372. http://dx.doi.org/10.1111/ j.1911-3846.2012.01193.x

Aggarwal, R., Klapper, L. y Wysocki, P. P. (2005). Portfolio preferences of foreign institutional investors. Journal of Banking \& Finance, 29, 2919-2946. http://dx.doi.org/10.1016/j.jbankfin.2004.09.008

Agostino, M., Drago, D. y Silipo, D. (2011). The value relevance of IFRS in the European banking industry. Review of Quantitative Finance and Accounting, 36(3), 437-457. http://dx.doi.org/10.1007/s11156-010-0184-1

Armstrong, C., Barth, M. y Riedl, E. (2010). Market reaction to the adoption of IFRS in Europe. The Accounting Review, 85, 31-61. http://dx.doi.org/10.2308/accr.2010.85.1.31

Ball, R. (2006). International Financial Reporting Standards (IFRS): Pros and cons for investors. Accounting and Bussines Research, 36, 5-27. http://dx.doi.org/10.1080/00014788.2006.9730040

Ball, R., Robin, A. y Wu, J. S. (2003). Incentives versus standards: properties of accounting income in four East Asian countries, and implications for acceptance of IAS. Journal of Accounting and Economics, 36(1-3), 235-270. http://dx.doi.org/10.1016/j.jacceco.2003.10.003

Ball, R. y Shivakumar, L. (2005). Earnings quality in UK private firms: Comparative loss recognition timeliness. Journal of Accounting and Economics, 39(1), 83-128. http://dx.doi.org/10.1016/j.jacceco.2004.04.001

Barth, M.E., Landsman, W.R., Lang, M. y Williams, C. (2007), Accounting quality: International Accounting Standards and US GAAP, Working paper, Stanford University, University of North Carolina, Stanford.

Barth, M. E., Landsman, W. R. y Lang, M. H. (2008). International accounting standards and accounting quality. Journal of Accounting Research, 46(3), 467-498. http://dx.doi.org/10.1111/j.1475-679x.2008.00287.x

Bartov, E., Goldberg, S. R. y Kim, M. (2005). Comparative value relevance among German, U.S. and International Accounting Standards: A German stock market perspective. Journal of Accounting Auditing and Finance, 20(2), 95-119. http://dx.doi.org/10.2139/ssrn.316525

Bilgic, F. y Ibis, C. (2013). Effects of new financial reporting standards on value relevance - A study about Turkish stock markets. International Journal of Economics and Finance, 5(10), 126-140. http://dx.doi.org/10.5539/ijef.v5n10p126

Burgstahler, D., Hail, L. y Leuz, C. (2006). The importance of reporting incentives: Earnings management in European private and public firms. The Accounting Review, 81(5), 983-1016. http://dx.doi.org/10.2308/accr.2006.81.5.983

Cameran, M., Campa, D. y Pettinicchio, A. (2014). IFRS Adoption among private companies: Impact on earnings quality. Journal of Accounting, Auditing \& Finance, 29(3), 278-305. http://dx.doi.org/10.1177/0148558x14534260

Chen, H., Tang, Q., Jiang, Y. y Lin, Z. (2010). The role of international financial reporting standards in accounting quality: Evidence from the European Union. Journal of International Financial Management \& Accounting, 21(3), $220-278$. http://dx.doi.org/10.1111/j.1467-646x.2010.01041.x 
Davis-Friday, P. Y. y Gordon, E. A. (2002). The effect of macroeconomic changes on the value relevance of accounting information: The case of Mexico and the 1995 financial crisis. Indiana: University of Notre Dame y Rutgers University. http://dx.doi.org/10.2139/ssrn.322762

Davis-Friday, P. Y. y Rivera, J. M. (2000). Inflation accounting and 20-F disclosures: Evidence from Mexico. Accounting Horizons, 14(2), 113-135. http://dx.doi.org/10.2308/acch.2000.14.2.113

Daske, H., Hail, L., Leuz, C. y Verdi, R. (2007). Adopting a label: Heterogeneity in the economic consequences of IFRS adoptions. In Working paper. University of Pennsylvania and University of Chicago. http://dx.doi.org/10.1111/ 1475-679x.12005

Daske, H., Hail, L., Leuz, C. y Verdi, R. (2008). Mandatory IFRS reporting around the world: Early evidence on the economic consequences. Journal of Accounting Research, 46(5), 1085-1142. http://dx.doi.org/10.1111/ j.1475-679x.2008.00306.x

Dechow, P. M., Ge, W. y Schrand, K. (2010). Understanding earnings quality: A review of the proxies, their determinants and their consequences. Journal of Accounting and Economics, 50(2-3), 344-401. http://dx.doi.org/ 10.1016/j.jacceco.2010.09.001

Dechow, P. M. y Schrand, K. (2004). Earnings quality. Charlotteville, VA: Research Foundation Publications.

Dorantes, C. (2013). The relevance of using accounting fundamentals in the Mexican stock market. Journal of Economics, Finance and Administrative Science, 18(Special Issue), 2-10. http://dx.doi.org/10.1016/s2077-1886(13)70024-6

Dumitru, G. (2011). The accounting information quality concept. Journal of Academic Research in Economics, 3(3), $559-569$

Fama, E. (1970). Efficient capital markets: A review of theory and empirical work. The Journal of Finance, 25 (2), 383-417. http://dx.doi.org/10.2307/2325486

Francis, J., LaFond, R., Olsson, P. M. y Schipper, K. (2004). Costs of equity and earnings attributes. The Accounting Review, 79(4), 967-1010. http://dx.doi.org/10.2308/accr.2004.79.4.967

Hribar, P., Kravel, T. y Wilson, R. (2014). A new measure of accounting quality. Review of Accounting Studies, 19, 506-538. http://dx.doi.org/10.1007/s11142-013-9253-8

Hung, M. y Subramanyam, K. (2007). Financial statement effects of adopting international accounting standards: The case of Germany. Review of Accounting Studies, 12(4), 623-657. http://dx.doi.org/10.1007/s11142-007-9049-9

Jarva, H. y Lantto, A.-M. (2012). The information content of IFRS versus domestic accounting standards: Evidence from Finland. The Finnish Journal of Business Economics, 2, 141-177. http://dx.doi.org/10.2139/ssrn.1588087

Karğın, S. (2013). The impact of IFRS on the value relevance of accounting information: Evidence from Turkish firms. International Journal of Economics and Finance, 5(4), 71-80. http://dx.doi.org/10.5539/ijef.v5n4p71

Khan, M. y Watts, R. L. (2009). Estimation and empirical properties of a firm-year measure of accounting conservatism. Journal of Accounting and Economics, 48(2-3), 132-150. http://dx.doi.org/10.1016/j.jacceco.2009.08.002

Kothari, S. P. y Zimmerman, J. L. (1995). Price and return models. Journal of Accounting and Economics, 20(2), 155-192. http://dx.doi.org/10.1016/0165-4101(95)00399-4

Lang, M., Smith Raedy, J. y Wilson, W. (2006). Earnings management and cross listing: Are reconciled earnings comparable to US earnings? Journal of Accounting and Economics, 42(1-2), 255-283. http://dx.doi.org/10.1016/ j.jacceco.2006.04.005

Mohan, N. y John, F. (2011). Value relevance of accounting information - An Indian perspective. Journal of Finance, Accounting and Management, 2(1), 16-26.

Morais, A. I. y Curto, D. J. (2008). Accounting quality and the adoption of IASB standards. Portuguese evidence. Revista Contabilidade and Finanças, 19(48), 103-111. http://dx.doi.org/10.1590/s1519-70772008000300009

Nair, R. D. y Frank, W. G. (1980). The impact of disclosure and measurement practices on international accounting classifications. The Accounting Review, 55, 426-450. http://dx.doi.org/10.1016/b978-0-408-10841-6.50010-5

Nikolaev, V. V. (2010). Debt covenants and accounting conservatism. Journal of Accounting Research, 48(1), 51-89. http://dx.doi.org/10.1111/j.1475-679x.2009.00359.x

Ohlson, J. A. (1995). Earnings, book values, and dividends in equity valuation. Contemporary Accounting Research, 11(2), 661-687. http://dx.doi.org/10.1111/j.1911-3846.1995.tb00461.x

Paananen, M. y Lin, H. (2009). the development of accounting quality of IAS and IFRS over time: The case of Germany. Journal of International Accounting Research, 8(1), 31-55. http://dx.doi.org/10.2308/jiar.2009.8.1.31

Penman, S. y Zhang, X. (2002). Accounting conservatism, the quality of earnings, and stock returns,. The Accounting Review, 77(2), 237-264. http://dx.doi.org/10.2308/accr.2002.77.2.237

Rawashdeh, M. (2003). Effects of introducing international accounting standards on Amman stock exchange. Journal of American Academy of Business, 3(1), 361-366.

Schipper, K. (2003). Principles-based accounting standards. Accounting Horizons, 17, 61-72. http://dx.doi.org/10.2308/ acch.2003.17.1.61 
Standifird, S. S. y Weinstein, M. (2002). Establishing legitimacy in emerging markets: An empirical comparison of the Warsaw, Budapest, and Prague stock exchanges. Journal of Comparative Policy Analysis, 4(2), $143-163$. http://dx.doi.org/10.1080/13876980208412676

Tsalavoutas, I., André, P. y Evans, L. (2010). Transition to IFRS and value relevance in a small but developed market: A look at Greek evidence, working paper. Stirling: University of Stirling y ESSEC Business School. Marzo 1.

Watts, R. L. (2003). Conservatism in accounting. Part II: Evidence and research opportunities. Accounting Horizons, 17(4), 287-301. http://dx.doi.org/10.2308/acch.2003.17.4.287 